\title{
The effects of vibration foam roller applied to hamstring on the quadriceps electromyography activity and hamstring flexibility
}

\author{
Jae-Heon Lim', Chi-Bok Park ${ }^{2 *}$, Byeong-Geun Kim³ \\ 'Department of Physical Therapy, Wonkwang Health Science University, Iksan, Korea \\ 2Department of Physical Therapy, Somoonnan Orthopedic Clinic, Gwangju, Korea \\ ${ }^{3}$ Department of Integrated Medicine, Graduate School, Nambu University, Gwangju, Korea
}

This study investigates the effect of vibration foam roller (VFR) applied to the hamstring, on the quadriceps electromyography (EMG) activity and hamstring flexibility. A total of 16 subjects were randomly assigned to either the VFR group $(n=8)$ or the nonvibration foam roller group (NVFR, $n=8$ ). The two groups applied the foam roller or the VFR to the dominant hamstring, at a rate of 40 times per min, for a total of 200 times in $5 \mathrm{~min}$. The primary outcome was to measure hamstring extensibility using the sit and reach test. The EMG activity of the dominant rectus femoris (RF), vastus lateralis (VL), and vastus medialis (VM) was measured as the secondary outcome. Results indicate a significant increase in the sit and reach distance after the intervention in both groups, as compared to preintervention. The sit and reach difference between VFR and NVFR show no significant difference after the inter- vention. Significant increases were observed after intervention in the \%maximum voluntary isometric contraction (\%MVIC) of VL, VM, and RF in the VFR group, and RF in the NVFR group, as compared to the preintervention values. Furthermore, \%MVIC values of $\mathrm{VL}$ and $\mathrm{RF}$, but not the VM muscle, were significantly different between the two groups. These findings suggest that regardless of vibration, the application of foam roller to hamstring may exert a positive effect on hamstring flexibility. Furthermore, these results indicate that the application of foam roller with vibrations causes more EMG activation in the antagonists.

Keywords: Vibration foam roller, Electromyography, Hamstring flexibility, Antagonist

\section{INTRODUCTION}

Along with the quadriceps, hamstring contribute to stable knee movement and play a significant role in various physical activities such as walking, running, and jumping. Adaptive shortening occurs more easily in the hamstring than in other muscles, resulting in loss of hip bone curvature and spine alignment (López-Miñarro et al., 2012). Misalignment reduces the neuromuscular efficiency and range of motion, increases energy expenditure, and is more likely to put greater strain on the knee.

Methods for resolving shortening of the hamstring causing knee injuries include rolfing, connective tissue massage, and muscle energy technique. Foam roller, which are particularly useful for the relaxation of myofascia, serve to apply pressure by using the limb weight of the patient; this technique has been in the spotlight recently due to low treatment cost (Wiewelhove et al., 2019). Further researches on the effect of foam roller are ongoing. Several studies show that foam roller increase the range of motion, improve neuromuscular function, reduce muscle, myofascia pain and convulsion, and regulate muscle imbalance. This is reportedly an outcome of stimulating the mechanical receptors, and changing the muscle and myofascia. The most direct effect is due to the thixotropic properties of the myofascia with increased range of motion. The heat generated by mechanical friction between the foam roller and the body increases fluidity of the muscles and myofascia component, allowing for an increased range of motion. It is

\footnotetext{
*Corresponding author: Chi-Bok Park (D) https://orcid.org/0000-0003-3791-7966 Department of Physical Therapy, Somoonnan Orthopedic Clinic,

39 Pungamjungang-ro, Seo-gu, Gwangju 62058, Korea

E-mail: oasislovept@hanmail.net

Received: May 6, 2019 / Accepted: June 14, 2019
} This is an Open Access article distributed under the terms of the Creative Commons At-
tribution Non-Commercial License (http://creativecommons.org/licenses/by-nc/4.0/)
which permits unrestricted non-commercial use, distribution, and reproduction in any
medium, provided the original work is properly cited. 
suggested that the application of foam roller to the hamstring is more useful for increasing the range of motion than other stretching methods (Madoni et al., 2018). It is also reported that compared to other stretching methods, the foam roller has an immediate effect on improving range of motion of the knee with respect to bending and increasing extension angle of the knee (Su et al., 2017).

However, to relax the muscles and myofascia to the maximum, a continuous and rhythmic foam roller needs to be applied. This is often associated with the disadvantage of difficulty in using the limbs for an extended period of time. Recently, foam roller combining continuous vibration have been commercialized. Improved range of knee motion has been reported after applying vibration with the foam roller to the knee flexor muscles, as compared with using a regular foam roller without vibration (Lee et al., 2018). In addition, the vibrating foam roller is also reported to improve the range of motion of the knee flexion joints (Cheatham et al., 2017). Taken together, foam roller with vibration are more effective than those without vibration, with respect to increasing the range of motion.

Increase in the range of motion is explained by various factors, including the thixotropic properties mentioned above and the degree of activation of the nerve root. The study reporting that a foam roller applied on the hamstring affects a decrease of electromyography (EMG) activity was explained by other mechanisms. Therefore, further research is required to better determine the effect of foam roller with respect to the agonistic muscle and antagonistic muscle. Additionally, the differences due to vibration have not yet been fully elucidated thus far.

The primary purpose of this study was to determine if use of vibration foam roller increases flexibility of the hamstring muscle group. The secondary purpose of this study was to investigate how the application of foam roller to the hamstring affects the EMG activity of the antagonistic muscle quadriceps, and to show any differences due to vibrations. We hypothesized that foam rolling with vibration would result in greater increases in hamstring flexibility. Furthermore, we expected significant differences between vibration foam rolling and nonvibration foam rolling.

\section{MATERIALS AND METHODS}

\section{Subjects}

The present study involved 16 recreationally active persons. Setting the statistical power, effect size, statistical significance level to $80 \%, 1.6$, and $\alpha=0.05$, respectively, via a 1:1 matching of the two groups as a condition, we determined the number of subjects required was 16 persons (Madoni et al., 2018). Participants without neurological or orthopedic disorders that could affect interventions, and those who could not apply vibration stimuli were selected. All subjects were fully informed about the purpose and procedure of the study, in accordance with the ethical standards of the Declaration of Helsinki; only those who voluntarily signed the study participation agreement were enrolled as subjects. Apparently obese subjects were excluded from this study, since we believed that excessive body covering fat may negatively affect the foam roller on myofascial. Subjects were asked to avoid drinking alcohol 2 days prior to this experiment. Subjects were requested to put on the same footwear for all sessions.

\section{Study design}

This study was based on the pretest-posttest comparison group design. Participants were divided into two groups: the vibration foam roller (VFR) group $(\mathrm{n}=8)$ and the nonvibration foam roller (NVFR) group $(\mathrm{n}=8)$, by drawing lots for randomization. All procedures were performed by an examiner who had never participated in this intervention. The primary outcome was identified as the sit and reach test, and the secondary outcome was determined as $\mathrm{EMG}$ of the quadriceps.

\section{Foam roller technique}

The application of the foam roller method was as follows. Both groups sat on the floor with the non-dominant leg flexed as the stabilizer and the dominant leg extended. The foam roller was positioned perpendicular to the hamstring muscle of the dominant leg. Using both hands, subjects were asked to lift their hips and move forward and backward. The hands remained fixed on the floor and did not move during the foam rolling application. Both applications involved rolling only the hamstring located between the gluteal fold and popliteal fossa. Subjects were instructed to keep maximum weight above the dominant leg. The cycle comprised of 40 movements per minute, for a total of 200 movements in $5 \mathrm{~min}$; after every minute, subjects were rested for $30 \mathrm{sec}$. The VFR performed the above procedure using a vibrating tool, which was lacking in the NVFR. A vibration of $32 \mathrm{~Hz}$ was applied using the VFR (Hyperice, VYPER 2.0, Irvine, CA, USA). The same instrument was used as the NVFR, by turning off the vibration application. The foam roller dimensions were $30 \mathrm{~cm}$ in length and $15 \mathrm{~cm}$ in diameter. The stop watch used to check a time foam roller application and resting bout. 


\section{EMG (\%MVIC) activity}

An 8-EMG (FREEEMG, BTS S.P.A., Milano, Italy) was applied to measure the muscle activity of quadriceps, with sampling rate of 1,000 Hz and band pass filter 20-500 Hz. Data were collected for $5 \mathrm{sec}$; to reduce the error of the data value, the data was measured for only $3 \mathrm{sec}$, excluding the first and last $1 \mathrm{sec}$. After calculating root mean square values of the raw knee extensor EMG, all data were processed for normalizing to the maximum voluntary isometric contraction (MVIC). The mean value of the EMG data is represented as a percentage MVIC. To achieve the best EMG signal conductivity while reducing noise as much as possible, all skin hairs were removed, and the defoliated area was sterilized with alcohol before attaching the electrodes. The surface-EMG signal of the dominant leg was set by requesting the participant to kick a ball, and was recorded via electrodes placed on the rectus femoris (RF), vastus lateralis (VL), vastus medialis (VM). Electromyographic (EMG) activity of the VL, VM, and RF was monitored during active knee extension in the sitting position. The electromyogram heads were attached as follows: at the dominant $\mathrm{RF}$, half-way anterior inferior iliac spine from superior part of the patella, 9 to $16 \mathrm{~cm}$ superior to patella; at the dominant VM, 3 to $4 \mathrm{~cm}$ medial to the superomedial patella border, orientated $\sim 55^{\circ}$ to the vertical; at the dominant VL, 6 to $8 \mathrm{~cm}$ lateral to the superior border of the patella, orientated $\sim 15^{\circ}$ to the vertical (Rainoldi et al., 2004).

\section{Sit and reach test}

Hamstring flexibility was performed using the sit and reach test. The participants sat on the floor with dominant leg extended and the other leg kept flexed. Bare feet were positioned flat against a box, fixing their foot at $90^{\circ}$ angle to the floor. The experimenter continued extending the dominant knee on the floor. With one's hands on the other with hands, and keep subject's palms facing the floor, they reached forward along sit and reach instrument. The subjects were asked to stop if they felt any discomfort or stiffness on the posterior thigh. This position was maintained for $5 \mathrm{sec}$, and the distance covered was recorded in centimeter. Two measurements were performed at the pre- and postintervention, and the highest score was used for final data analysis. The validity of the sit and reach test has previously been corroborated for estimating hamstring flexibility $(r=0.46-0.67)$ (Mayorga-Vega et al., 2014), and has a high-reliability estimate $(0.96<\mathrm{R}<0.99)$ (Jackson and Baker, 1986; Shaulis et al., 1994).
Table 1. General characteristics of participants

\begin{tabular}{lcccc}
\hline Characteristic & VFR & NVFR & T-value & $P$-value \\
\hline Gender, male:female & $5: 3$ & $6: 2$ & - & - \\
Dominant, left:right & $3: 5$ & $1: 7$ & - & - \\
Age $(\mathrm{yr})$ & $20.37 \pm 1.06$ & $20.75 \pm 1.39$ & -0.607 & 0.554 \\
Height $(\mathrm{cm})$ & $168.00 \pm 9.41$ & $171.63 \pm 7.09$ & -0.870 & 0.399 \\
Weight $(\mathrm{kg})$ & $59.88 \pm 8.79$ & $67.25 \pm 14.93$ & -1.204 & 0.248 \\
BMI $\left(\mathrm{kg} / \mathrm{m}^{2}\right)$ & $20.85 \pm 1.48$ & $22.68 \pm 4.19$ & -1.170 & 0.262 \\
\hline
\end{tabular}

Values are presented as mean \pm standard deviation.

VFR, vibration foam roller; NVFR, nonvibration foam roller; BMl, body mass index.

\section{Data analysis}

All data were analyzed using the IBM SPSS Statistics ver. 21.0 (IBM Co., Armonk, NY, USA). The general characteristics of the subjects are expressed as the mean \pm standard deviation, except for gender and dominant leg. The Shapiro-Wilks test was used to verify the normality. Independent $t$-test examined differences in the general characteristics of the participants between the two groups. A paired $t$-test was used to determine the difference between the groups before and after the intervention, and an independent $t$-test was used to examine the differences between the two groups. Statistical significance was set at 0.05 .

\section{RESULTS}

\section{General characteristics of subjects}

A total of 16 subjects were included in this study. Each group was allocated eight subjects. General characteristics of the participants (age, height, body weight, body mass index) were significantly not different between the two groups $(P>0.05)$, as presented in Table 1.

\section{Primary outcome}

The primary outcome measured the hamstring extensibility by applying the sit and reach test. The sit and reach distance after intervention in both groups was significantly increased than preintervention (FRV group: $t=2.987, P=0.02$; NFRV group: $t=$ 3.424, $P=0.011)(P<0.05)$. The sit and reach difference between VFR and NVFR showed no significant difference after intervention $(t=-0.949, P=0.359)$ (Fig. 1).

\section{Secondary outcome}

The secondary outcome measured the EMG activity of the VL, $\mathrm{VM}$, and RF during active knee extension in sitting. EMG data were normalized to the \%MVIC. The \%MVIC of VL, VM, and $\mathrm{RF}$ after the intervention in the VFR group was significantly in- 


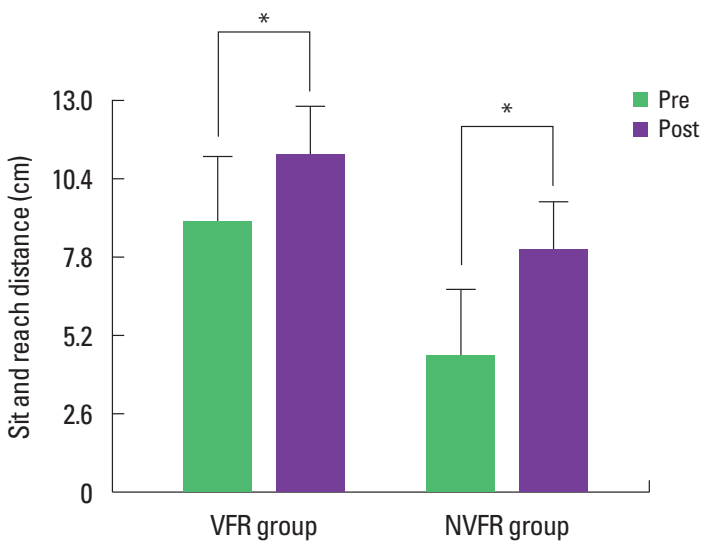

Fig. 1. The comparison of sit and reach distance between VFR and NVFR. VFR, vibration foam roller; NVFR, nonvibration foam roller. ${ }^{*} P<0.05$.

Table 2. The comparison of \%MVIC in each of the groups at pre- and posttest

\begin{tabular}{lccccc}
\hline Variable & Pretest & Posttest & $\begin{array}{c}\text { Difference } \\
\text { value }\end{array}$ & $T$-value & $P$-value \\
\hline Rectus femoris & & & & & \\
VFR & $15.12 \pm 7.09$ & $20.79 \pm 8.25$ & $5.67 \pm 4.23$ & 3.786 & 0.007 \\
NVFR & $16.76 \pm 7.39$ & $19.42 \pm 8.96$ & $2.67 \pm 2.08$ & 3.631 & 0.008 \\
$T$-value & -0.453 & & 2.781 & & \\
$P$-value & 0.657 & & 0.015 & & \\
Vastus lateralis & & & & & \\
VFR & $16.79 \pm 6.65$ & $20.93 \pm 6.16$ & $4.14 \pm 2.84$ & 4.120 & 0.004 \\
NVFR & $17.15 \pm 5.75$ & $16.20 \pm 6.50$ & $-0.95 \pm 1.81$ & -1.476 & 0.184 \\
$T$-value & -0.116 & & 4.267 & & \\
$P$-value & 0.909 & & 0.001 & & \\
Vastus medialis & & & & & \\
VFR & $14.28 \pm 5.50$ & $19.24 \pm 9.03$ & $4.96 \pm 5.84$ & 2.402 & 0.047 \\
NVFR & $17.81 \pm 7.38$ & $18.08 \pm 6.11$ & $0.26 \pm 4.23$ & 0.175 & 0.866 \\
$T$-value & -1.085 & & 1.843 & & \\
$P$-value & 0.296 & & 0.087 & & \\
\hline
\end{tabular}

Values are presented as mean \pm standard deviation.

$\% \mathrm{MVIC}$, \%maximum voluntary isometric contraction; VFR, vibration foam roller; NVFR, nonvibration foam roller.

creased, as compared with preintervention values. Similarly, \%MVIC of RF after the intervention in the NVFR group showed a significant increase as compared to preintervention values. Furthermore, except for the VM muscle, \%MVIC values in VL and $\mathrm{RF}$ were significantly different between the two groups (Table 2).

\section{DISCUSSION}

Applying a foam roller causes an increased range of motion and altered nerve-muscle activity (Wiewelhove et al., 2019). The primary aim of this study was to determine if the use of foam roller with vibration increases the flexibility of the hamstring muscle group. Since Couture et al. (2015) suggested that a total application time of more than 2 min of foam rolling is required to improve the hamstring flexibility, we applied a total of $5 \mathrm{~min}$ in this study. The foam roller was applied to the hamstring to investigate the effect of the foam roller on the agonistic muscle and antagonistic muscle. The secondary aim of this study was to investigate effect on the muscle through EMG activity of the quadriceps after application of the foam roller on hamstring.

Foam roller may be more useful for increasing flexibility without reducing the muscle strength ratio identified by the EMG activity of the hamstring and quadriceps, as compared with other stretches (Madoni et al., 2018). Application of both normal foam roller and VFRs to patients with hip joint pain revealed improved range of motion and reduced pain (Han et al., 2017). Another study that applied a foam roller for different durations reported that the application of a foam roller for $60 \mathrm{sec}$ to the quadriceps showed greater benefit than 20-sec application (Cavanaugh et al., 2017).

As determined by primary outcomes, the application of both VFR and NVFR methods to the hamstring area induced a significant improvement in hamstring flexibility. Regardless of vibration or nonvibration, the application of foam roller showed improved hamstring flexibility. This finding is consistent with a previous study, in which FR application to hamstring affected the hamstring flexibility, as examined by the sit and reach test (Peacock et al., 2015; Sullivan et al., 2013). Peacock et al. (2015) determined the effects of foam roller at the anteroposterior portion of major joints of the body in mediolateral axis on hamstring flexibility using the sit and reach test. They reported that foam roller had a significantly greater effect on increasing the sit and reach distance. Han et al. (2017) suggested that foam roller helped to increase the range of motion by increasing the extension and flexion of the hip joint.

The reason for increased hamstring flexibility is that the application of the foam roller reduces perceived muscle pain, and increases the pain pressure threshold. The range of motion can be attributed to decreased stiffness of the muscles (Pearcey et al., 2015). de Souza et al. (2019) suggested that constant pressure on the soft tissue through the foam roller suppresses the pain sensation and increases stretch tolerance through the pain-modulatory system.

Another reason for the effects of FR on hamstring flexibility would be ascribed to the changed viscoelastic and thixotropic properties of the fascia. The foam roller increases the muscular temperature and blood circulation due to the friction between 
connective tissue and a foam roller (Cheatham et al., 2015). Furthermore, the foam roller reduces muscle spasms, stimulates a deep muscle to stabilize nerves, and improves blood circulation through muscle relaxation (Cheatham et al., 2015). Hence, the myofascial, which is comprised of a gelatinous colloidal substance, would convert to a fluid form when exposed to heat and mechanical stress (de Souza et al., 2019). In addition, the foam roller was applied by back and forth movements, with the hands fixed to the floor in the long-sitting position. The sit and reach test to measure hamstring flexibility was also in the long-sitting position. The similarity between the position of test and intervention may be a factor that improves the actual sit and reach test (Wolpert et al., 2011).

Results from the secondary outcome reveal that foam roller with vibration increase the muscle activity of quadriceps, more than general foam roller, especially in muscles of RF and VL. It is thought that autogenic inhibition is well developed in the hamstring because the foam roller improves the muscle adhesions of the connective tissue of the hamstring. With autogenic inhibition mechanisms, the relaxation of the agonistic muscle through foam roller application is believed to increase the muscle activity of the quadriceps. When a foam roller applies pressure on the muscle tissue, it is known that the Golgi tendon organ (GTO) sends constant tension to the spinal cord to relax the muscles (Moore, 1984). In particular, the GTO is more sensitive to tension than stretch (Edin and Vallbo, 1990). The generation of tension through vibrations is thought to facilitate relaxation of the hamstring.

However, there have been some contradicting studies. Cavanaugh et al. (2017) reported that after the application of a foam roller to the quadriceps, the EMG activity of the biceps femoris was decreased. The author reported that the foam roller applied to the hamstring did not affect the muscular activity of quadriceps. They believe that the reason for decreased EMG activity of the opposing muscles with a foam roller may be related to the pain pressure threshold. Other authors have reported that the foam roller applied to the plantar flexors increases the pain pressure threshold and acutely decreases the pain perception, thereby increasing the amount of muscle movement more smoothly (Aboodarda et al., 2015). However, in the current study, it is considered that the effects of the pain pressure threshold are not realized, since the foam roller was applied smoothly at a rate that did not cause pain. Moreover, the VFR helped to improve the EMG activity. When vibration is applied to the muscles, contractions occur in the muscles to which the vibration is applied due to the appearance of tonic vibration reflex, which stimulates the sensory nerves through vibrations, causing reflex muscle contractions through alpha motor neuromuscular fibers (Dallas et al., 2015). This induces a reflexive contraction and consequently causes relaxation.

Bradbury-Squires et al. (2015) applied a foam roller to agonistic muscle and examined the effect on the antagonistic muscle. In the study, the 60-sec conditions applied to quadriceps were higher than activation for $20 \mathrm{sec}$. Since quadriceps and hamstring legs are responsible for stabilizing the knee, cocontraction of muscles is important to stabilize the joints. Therefore, it is thought that the frequency and time of continuous application for $1 \mathrm{~min}$ also affect the EMG activity. If the agonist and antagonist are unable to work effectively together, it is thought that foam roller could be a useful tool to change simultaneous contraction.

A vibration application study reported that vibration stimulates the muscles to help them perform better pain reduction and motor performance (Kasai et al., 1994). However, the reason for limiting the EMG difference according to vibration in this study is thought to be the vibration frequency range. A single frequency vibrating foam roller was applied three times a week for $20 \mathrm{~min}$ each, for a duration of 4 weeks (Han et al., 2017). In the comparison between VFR and simple foam roller, an increase was observed only in the iliotibial tract of the VFR group. Additionally, some authors conducted a 2-min mediation regimen between foam roller and a single frequency vibrating foam roller, but reported no significant difference in the range of motion of the knee joint (Cheatham et al., 2017). Therefore, it is considered necessary to apply different frequencies when applying a vibration stimulation, and also consider the application time. Future research is needed on various frequencies and application times for vibrating foam roller.

The limitations of this study are that it is difficult to generalize to normal adults, and only the immediate effects are examined. There is a need to continue the studies that diversify subjects and examine the long-term effects. Based on the results of this study, although the application of a foam roller to the hamstring helps to increase the EMG activity of quadriceps and flexibility of hamstring, VFRs are beneficial in overcoming limitations. If therapists want to relax the hamstring and change coactivation of knee joint, it will be helpful to apply a foam roller to the hamstring. Further studies are required to elucidate the extent and variety of neural responses to VFRs. Findings from the current study indicate that rolling the hamstring affects quadriceps activation. Given these results, changes in muscle activation following vibration foam rolling need to be recognized. 


\section{CONFLICT OF INTEREST}

No potential conflict of interest relevant to this article was reported.

\section{ACKNOWLEDGMENTS}

This paper was supported by Wonkwang Health Science University in 2019.

\section{REFERENCES}

Aboodarda SJ, Spence AJ, Button DC. Pain pressure threshold of a muscle tender spot increases following local and non-local rolling massage. BMC Musculoskelet Disord 2015;16:265.

Bradbury-Squires DJ, Noftall JC, Sullivan KM, Behm DG, Power KE, Button DC. Roller-massager application to the quadriceps and knee-joint range of motion and neuromuscular efficiency during a lunge. J Athl Train 2015;50:133-140.

Cavanaugh MT, Aboodarda SJ, Hodgson DD, Behm DG. Foam rolling of quadriceps decreases biceps femoris activation. J Strength Cond Res 2017;31:2238-2245.

Cheatham SW, Kolber MJ, Cain M, Lee M. The effects of self-myofascial release using a foam roll or roller massager on joint range of motion, muscle recovery, and performance: a systematic review. Int J Sports Phys Ther 2015;10:827-838.

Cheatham SW, Stull KR, Kolber MJ. Comparison of a vibrating foam roller and a non-vibrating foam roller intervention on knee range of motion and pressure pain threshold: A randomized controlled trial. J Sport Rehabil 2017;28:474-482.

Couture G, Karlik D, Glass SC, Hatzel BM. The effect of foam rolling duration on hamstring range of motion. Open Orthop J 2015;9:450-455.

Dallas G, Paradisis G, Kirialanis P, Mellos V, Argitaki P, Smirniotou A. The acute effects of different training loads of whole body vibration on flexibility and explosive strength of lower limbs in divers. Biol Sport 2015;32:235-241.

de Souza A, Sanchotene CG, Lopes CMDS, Beck JA, da Silva ACK, Pereira SM, Ruschel C. Acute effect of 2 self-myofascial release protocols on hip and ankle range of motion. J Sport Rehabil 2019;28:159-164.

Edin BB, Vallbo AB. Classification of human muscle stretch receptor afferents: a Bayesian approach. J Neurophysiol 1990;63:1314-1322.

Han SW, Lee YS, Lee DJ. The influence of the vibration form roller exercise on the pains in the muscles around the hip joint and the joint performance. J Phys Ther Sci 2017;29:1844-1847.

Jackson AW, Baker AA. The relationship of the sit and reach test to criteri- on measures of hamstring and back flexibility in young females. Res Q Exerc Sport 1986;57:183-186.

Kasai T, Kawanishi M, Yahagi S. Effects of upper limb muscle vibration on human voluntary wrist flexion-extension movements. Percept Mot Skills 1994;78:43-47.

Lee CL, Chu IH, Lyu BJ, Chang WD, Chang NJ. Comparison of vibration rolling, nonvibration rolling, and static stretching as a warm-up exercise on flexibility, joint proprioception, muscle strength, and balance in young adults. J Sports Sci 2018;36:2575-2582.

López-Miñarro PA, Muyor JM, Belmonte F, Alacid F. Acute effects of hamstring stretching on sagittal spinal curvatures and pelvic tilt. J Hum Kinet 2012;31:69-78.

Madoni SN, Costa PB, Coburn JW, Galpin AJ. Effects of foam rolling on range of motion, peak torque, muscle activation, and the hamstringsto-quadriceps strength ratios. J Strength Cond Res 2018;32:1821-1830.

Mayorga-Vega D, Merino-Marban R, Viciana J. Criterion-related validity of sit-and-reach tests for estimating hamstring and lumbar extensibility: a meta-analysis. J Sports Sci Med 2014;13:1-14.

Moore JC. The Golgi tendon organ: a review and update. Am J Occup Ther 1984;38:227-236.

Peacock CA, Krein DD, Antonio J, Sanders GJ, Silver TA, Colas M. Comparing acute bouts of sagittal plane progression foam rolling vs. frontal plane progression foam rolling. J Strength Cond Res 2015;29:23102315.

Pearcey GE, Bradbury-Squires DJ, Kawamoto JE, Drinkwater EJ, Behm DG, Button DC. Foam rolling for delayed-onset muscle soreness and recovery of dynamic performance measures. J Athl Train 2015;50:5-13.

Rainoldi A, Melchiorri G, Caruso I. A method for positioning electrodes during surface EMG recordings in lower limb muscles. J Neurosci Methods 2004;134:37-43.

Shaulis D, Golding LA, Tandy RD. Reliability of the AAHPERD functional fitness assessment across multiple practice sessions in older men and women. J Aging Phys Act 1994;2:273-279.

Su H, Chang NJ, Wu WL, Guo LY, Chu IH. Acute effects of foam rolling, static stretching, and dynamic stretching during warm-ups on muscular flexibility and strength in young adults. J Sport Rehabil 2017;26:469-477.

Sullivan KM, Silvey DB, Button DC, Behm DG. Roller-massager application to the hamstrings increases sit-and-reach range of motion within five to ten seconds without performance impairments. Int J Sports Phys Ther 2013;8:228-236.

Wiewelhove T, Döweling A, Schneider C, Hottenrott L, Meyer T, Kellmann M, Pfeiffer M, Ferrauti A. A meta-analysis of the effects of foam rolling on performance and recovery. Front Physiol 2019;10:376.

Wolpert DM, Diedrichsen J, Flanagan JR. Principles of sensorimotor learning. Nat Rev Neurosci 2011;12:739-751. 